\title{
High strain rate deformation behavior of a continuous fiber reinforced aluminum metal matrix composite
}

\author{
M. Guden ${ }^{\mathrm{a}}$, I.W. Hall ${ }^{\mathrm{b}, *}$ \\ ${ }^{\mathrm{a}}$ Izmir Institute of Technology, Izmir, Turkey \\ ${ }^{\mathrm{b}}$ University of Delaware, Mechanical Engineering, Newark, DE 19716, USA
}

\begin{abstract}
An aluminum metal matrix composite reinforced with continuous unidirectional $\alpha-\mathrm{Al}_{2} \mathrm{O}_{3}$ fibers has been compression tested at quasi-static and dynamic strain rates. In the transverse direction, the composite showed increased flow stress and maximum stress within the studied strain rate regime, $10^{-3}$ to $3500 \mathrm{~s}^{-1}$. The strain rate sensitivity of the flow stress in this direction was found to be similar to that of a similar, but unreinforced, alloy determined from previous work. In the longitudinal direction, the maximum stress of the composite increased with increasing strain rate within the range $10^{-5}$ to $700 \mathrm{~s}^{-1}$. The strain rate dependent maximum stress in this direction was described by the strain rate dependent fiber buckling stress. (C) 2000 Elsevier Science Ltd. All rights reserved.
\end{abstract}

Keywords: Continuous fiber; Metal matrix composite; High strain rate; Compression

\section{Introduction}

The high strain rate mechanical behavior of Metal Matrix Composites (MMCs) and as well as other composites is important for several reasons. First, knowledge of the mechanical properties of composites at high strain rates is needed for designing with these materials in applications where sudden changes in loading rates are likely to occur. Second, knowledge of both the dynamic and quasi-static mechanical responses can be used to establish the constitutive equations which are necessary to increase the confidence limits of these materials, particularly if they are to be used in critical structural applications.

\footnotetext{
*Corresponding author. Tel.: +1-3028312062; fax: +13028314545 .

E-mail address: hall@me.udel.edu (I.W. Hall).
}

This study reports further research on the high strain rate compressive deformation behavior of a continuous fiber reinforced Al MMC tested in the longitudinal and transverse directions and compared with its quasi-static behavior. Previous work on this material [1] reported some preliminary mechanical property results. This report now presents further results of the quasi-static and dynamic compression tests and uses the data to examine the predictions of simple constitutive equations for unidirectionally reinforced composite.

\section{Materials and testing methods}

The $\mathrm{FP}^{\text {(ब) }}$ (99\% $\alpha$-alumina)/Al composite was produced by DuPont Company using a molten metal infiltration technique. The composite contained 35 volume percent $\left(V_{\mathrm{f}} \%\right)$ unidirectional fibers and $3 \% \mathrm{Li}$ in the 
Al matrix. The continuous FP fibers were $20 \mu \mathrm{m}$ in diameter.

The as-received composite was in the form of 15 $\mathrm{mm}$ thick plate and slices were cut normal and parallel to the fiber plane in order to core drill cylindrical specimens in orientations corresponding to longitudinal $\left(0^{\circ}\right)$ and transverse $\left(90^{\circ}\right)$ directions. Specimens tested in the transverse direction had an aspect ratio (length/ diameter) of 0.5 and a diameter of $8.2 \mathrm{~mm}$. The specimens tested in the longitudinal direction were $12 \mathrm{~mm}$ in length and $6.6 \mathrm{~mm}$ in diameter. Quasi-static and high strain rate compression tests were conducted using a screw-driven Instron and a Split Hopkinson Pressure Bar (SHPB), respectively. The striker, incident and transmitter bars of the SHPB apparatus are made of Inconel 718 alloy and are $19 \mathrm{~mm}$ in diameter and 724, 3658, $1440 \mathrm{~mm}$ long, respectively.

Reduction of raw data from the SHPB is based upon elastic wave propagation theory. A consequence of this is that there is considerable uncertainty in the acuracy of the data for the first few percent of strain during which time successive wave reflections occur and build up a state of homogeneous stress. It is, therefore, common practice to use a value of flow stress at $5 \%$ strain, for example, as a reproducible mechanical property parameter instead of yield stress. This is the approach used here. Further details of specimen preparation and testing methods are discussed in Ref. [1].

\section{Results}

\subsection{Transverse direction}

Typical stress/strain curves of the FP-Al reinforced MMC at different strain rates in the $90^{\circ}$ direction are

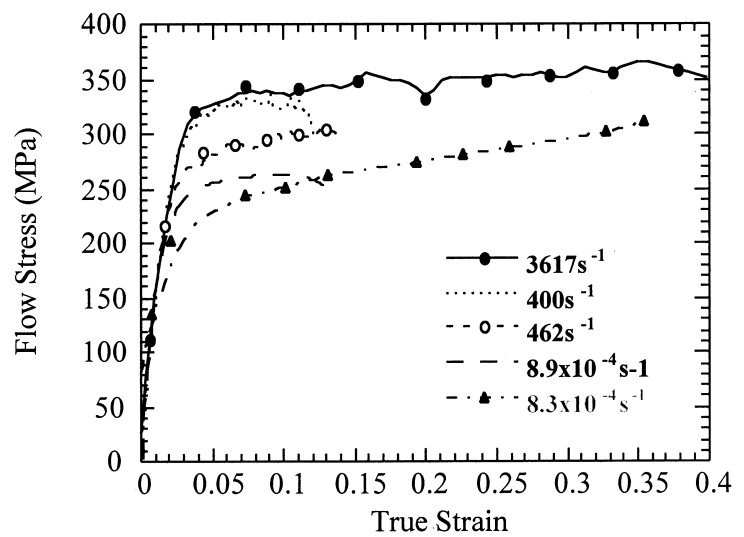

Fig. 1. Transverse stress/strain curves of FP reinforced $\mathrm{Al}$ $\mathrm{MMC}$ at different strain rates. shown in Fig. 1. The stress values, in common with quasi-static strain rates, were found to fluctuate significantly. At relatively low strain rates, $\sim 400 \mathrm{~s}^{-1}$, some of the samples failed early in the SHPB test showing stress maxima as shown in Fig. 1. At increasing strain rates, although no maximum peak stress was detected, the stress/strain curves reached a stress plateau such as shown in Fig. 1 at $3617 \mathrm{~s}^{-1}$. The stress corresponding to the stress plateau at higher strain rates, along with the peak stresses at lower strain rates, are referred to hereafter as maximum stress. The 5\% flow stress values at high strain rates are higher than those of quasi-static strain rates, as depicted in Fig. 1. In samples which failed early, the maximum stress also increased with strain rate, similar to flow stress.

In Fig. 2, the increase of 5\% flow stress and maximum stresses are shown as a function of the logarithm of strain rate. Despite quite large variations between individual data points at similar strain rates, the overall trend of the flow and maximum stresses is clearly to increase as the strain rate increases.

Failure in this direction occurred in a shear mode, at $45^{\circ}$ to the compression axis. In order to distinguish any possible differences in damage accumulation modes in the composite at quasi-static and dynamic strain rates, samples were tested until approximately the same strain at quasi-static and dynamic strain rates and then cut and metallographically prepared for microscopic observations. High strain rate testing to a specific strain was achieved by inserting the sample into a loose-fitting collar which prevented further deformation once it came into contact with the anvils of the SHPB: different collar lengths permitted different strain levels to be investigated. The comparison has been performed for samples tested at $10^{-3}$ and $400 \mathrm{~s}^{-1}$ until about $15 \%$ strain. The sample tested quasi-statically showed matrix and extensive fiber fracture in the plane at $45^{\circ}$ to the compression axis. In the sample tested dynamically the major damage was microcracks

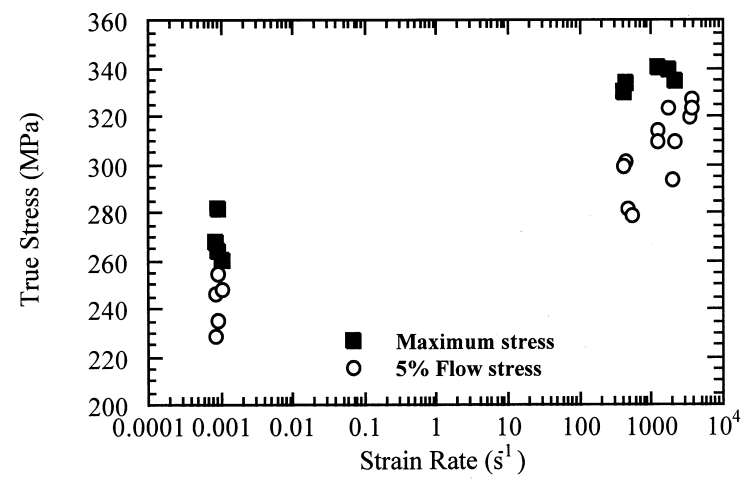

Fig. 2. Variation with strain rate of transverse maximum and flow (5\% strain) sresses of FP reinforced Al MMC. 
between fibers which might lead to debonding at later stages of deformations [1]. These observations proved that, at least at relatively low stress values, failure in samples tested quasi-statically had initiated at lower strain levels when compared to dynamically tested specimens.

\subsection{Longitudinal direction}

In the longitudinal direction, initial experiments were conducted using several different metal inserts ( $\sim 2 \mathrm{~mm}$ in thickness) between specimen and bar interfaces in order to (a) protect the faces of the Inconel bars and (b) prevent premature failure of the composite by brooming. The latter protection was provided by using metal inserts on the faces of the anvils, into which the composite sample's ends indented during testing. Several different metal inserts were tried including $\mathrm{Cu}$, steel and Al. Although placement of metal inserts successfully prevented failure by brooming, it could not give any tractable stress and strain data due to extensive plastic deformation of the inserts. Since quasi-static testing shows the stress-strain curve of the composite in the longitudinal direction to be non-linear, placing strain gages and calculating stress values from modulus and measured strain data was also impossible. Another method was to use constraining metal rings at the specimen ends. This method also failed due to the sliding of the rings on the surface of the specimen during compression testing. Finally tool steel end caps, containing a shallow recess of the same diameter as the specimens, were used to support the composite samples. The ends of the composite sample were tightly inserted into the caps with little pressure. These caps were impedance matched to the Inconel bar.

Several experiments were conducted by mounting

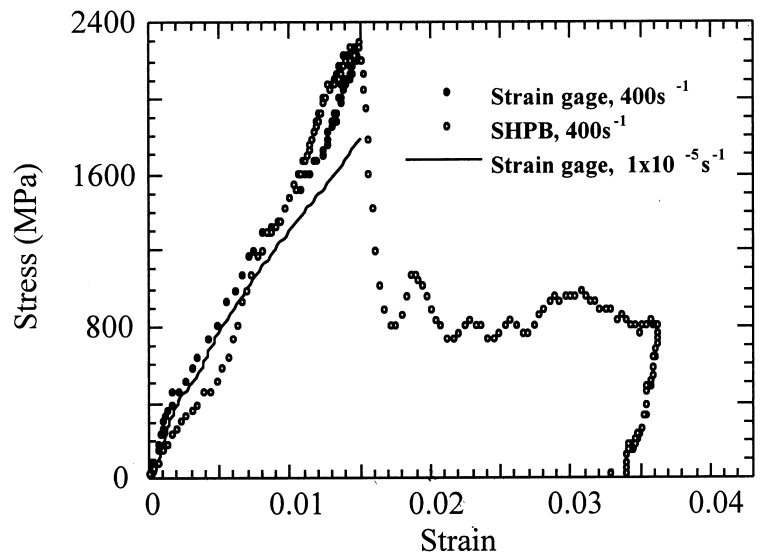

Fig. 3. Dynamic and quasi-static stress/strain curves of FP reinforced $\mathrm{Al}$. strain gages on the samples. Typical stress-strain curves of strain-gaged composite samples in the longitudinal direction at dynamic and quasi-static strain rates are shown in Fig. 3. It can be noted from Fig. 3 that the measured stress values from SHPB and straingaged samples are very close except, of course, at the initial small strains. At around $1.5 \%$ strain, the stress suddenly dropped to a lower value as shown in Fig. 3. This was due to localization of the deformation in the fiber buckled or kink region. The failure strains, corresponding to the previously mentioned maximum stresses are found to be around $1.5 \%$ at both quasi-static and dynamic strain rates. After $1.5 \%$ strain, the deformation was localized in the kink region but the sample still carried some load until the complete failure in the kink region.

In Fig. 4, failure stress is drawn as function of strain rate for both recessed and planar tool steel end caps tests. In the former tests, failure was due to kink formation as explained before and in the latter failure was due to brooming. As noted from Fig. 4, in both tests, the failure stress values increased as the strain rate increased. The stress values increased by $300-500$ MPa from quasi-static $\left(10^{-5} \mathrm{~s}^{-1}\right)$ to dynamic strain rates $\left(\sim 200-700 \mathrm{~s}^{-1}\right)$ on the average and confirms that the composite has strain rate sensitive failure stress within the studied strain rate range.

Of the two types of failure mechanism observed in this direction, failure from kink formation and by brooming, the former occurred when end caps or metal inserts were used to support the specimen ends and the latter occurred when the ends of the samples were not supported. In both failure modes the major damage formation leading to failure of the composite in compression was buckling of the fibers. However, failure by brooming occurred at relatively lower stresses, in the range $1500-1700 \mathrm{MPa}$. This is believed to be because of extensive plastic deformation of the

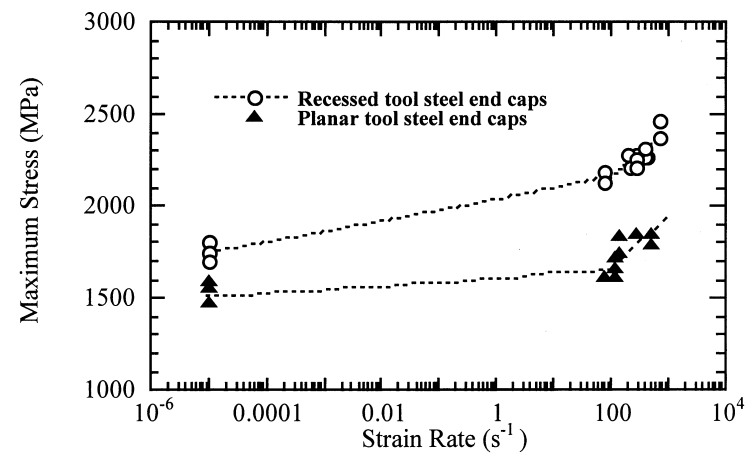

Fig. 4. Variation of the longitudinal maximum stresses of FP reinforced $\mathrm{Al} \mathrm{MMC}$ with strain rate. 

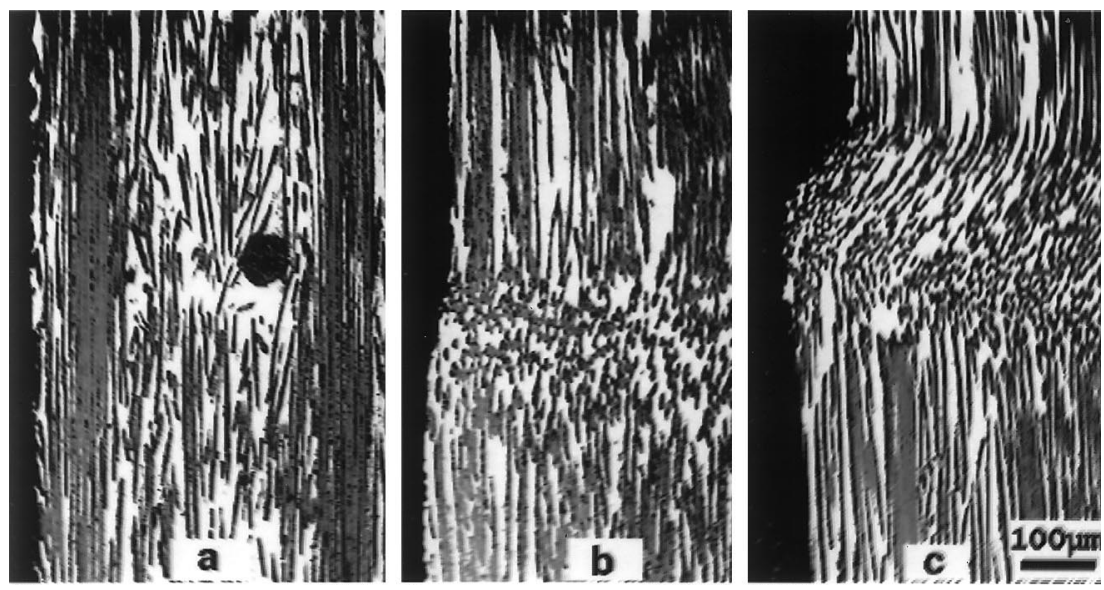

Fig. 5. Progressive kink formation (a) before kinking (1\% strain), (b) fiber rotation and buckling (1.8\% strain) and (c) developed kink region ( $3 \%$ strain).

matrix resulting from fiber buckling near to one of the unsupported specimen ends.

The consistent 300-500 MPa difference in test results between recessed and planar end caps illustrated in Fig. 4 clearly shows the desirability of standardizing test methods in this field. In this case, maximum stress is dependent upon failure mode and the failure mode is itself dependent upon precise test method.

The typical sequence of events during kink formation at dynamic strain rates is shown in Fig. 5. Specimens tested until $1,1.8$ and $3 \%$ strain were sectioned and observed by optical microscopy. These specimens were tested using 6061-T6 Al inserts. During testing, the inserts deformed together with the composite and the deformation was allowed to continue until 1, 1.8 and $3 \%$ strains in different specimens by sequentially increasing the striker bar velocity of the SHPB. It should be noted, therefore, that the micrographs of Fig. 5 shows only kink band evolution not the dependence of evolution on strain rate. The specimen tested until $1 \%$ strain showed no localized region of fiber buckling, but did show fiber misalignment in local regions which might be the precursor to kink formation, Fig. 5(a). At 1.8\% strain, the kink region has already been localized and fibers are rotated in the kink region, Fig. 5(b). At 3\% strain, the kink region was sharper and showed further fiber rotation as compared to $1.8 \%$ strain, (see Fig. 5(c)).

\section{Discussion}

In unidirectional composites, plastic microbuckling (a localized band of buckled fibers) is considered to be the primary failure mechanism limiting the composite strength in compression [2-5]. Experimental studies on carbon fiber reinforced epoxy composites have shown that microbuckling initiated from intentionally induced holes or notches [6-8]. The microbuckling was found to initiate from the edge of the hole at a stress level of $80 \%$ of the compressive strength and propagated through the specimen [6]. In unnotched specimens, microbuckling initiates in regions of fiber misalignment [9]. Fiber misaligned regions can result in local shearing of the matrix, resulting in a reduced axial strength which is about $20-30 \%$ of the stress required to buckle fibers in a perfectly aligned composite [10].

Several approaches to determining the composite microbuckling stress (usually considered as the maximum compressive strength) $\left(\sigma_{\mathrm{c}}\right)$ are tabulated in Table 1. Eq. (1) of Table 1, known as Rosen's Equation, considers only elastic buckling of the fibers and elastic shear of the matrix [11]. In Rosen's equation, $G$ is the shear modulus of the composite and $m$ refers to the matrix. Argon's equation [12] on the other hand considers a rigid perfectly plastic composite, with a yield strength of $\tau_{\mathrm{y}}$ and an initial fiber misalignment of $\phi$. Argon's equation essentially predicts a localized nucleus for fiber buckling which then propagates across the specimen forming macroscopic failure.

Table 1

Approaches for determining compressive strength of composites by microbuckling

\begin{tabular}{ll}
\hline Rosen [11] & $\sigma_{\mathrm{c}}=G_{\mathrm{m}} /\left(1-V_{\mathrm{f}}\right)=G$ \\
Argon [12] & $\sigma_{\mathrm{c}}=\tau_{\mathrm{y}} / \bar{\phi}$ \\
Budiansky [13] & $\sigma_{\mathrm{c}}=\tau_{\mathrm{y}}(\gamma) /(\bar{\phi}+\gamma)$
\end{tabular}


Budiansky [13] modified Argon's equation for elasticplastic shear response, Eq. (3) of Table 1. It can be easily seen from the formulae given in Table 1, that the dominant factors are the initial fiber misalignment and composite yield stress which scales with matrix shear yield strength. The interpolation of the compressive strength of the unidirectional polymeric composites to the matrix yield strength has shown a linear relationship [4]. This interpolation has further predicted an initial fiber misalignment of $3^{\circ}$ which seems reasonable for fiber lay-ups [14].

The above findings may also be applied to the FP reinforced MMC tested longitudinally at different strain rates. For that purpose, the rate sensitive flow stress behavior of the matrix and similar alloys have been analyzed. Typical similar alloys such as 8090 , 2091-Al and Al-2.76wt\%Li have been previously tested at different strain rates and found to have somewhat strain rate sensitive flow stress values $[15,16]$. The latter alloy, $\mathrm{Al}-2.76 \mathrm{wt} \% \mathrm{Li}$, is very similar to the present matrix alloy. The shear stress/strain behavior of the alloy was previously determined from torsion testing by Kobayashi [16] and, within the studied shear strain rates, the alloy has quite rate sensitive flow stresses. In Fig. 6, the equivalent compression stress/strain curves, converted from torsion tests, and transverse stress/ strain curves of the composite are shown together at different strain rates. Conversion of the torsional stress, strain and strain rate to the effective stress, strain and strain rate was carried out using the von Mises criterion. Although composite in the transverse direction exhibits higher stresses than the equivalent stress values of the $\mathrm{Al}-2.76 \mathrm{wt} \% \mathrm{Li}$ alloy, the increase in flow stresses with strain rate is quite similar, Fig. 6. The equivalent flow stress values of the $\mathrm{Al}-2.76 \mathrm{wt} \% \mathrm{Li}$

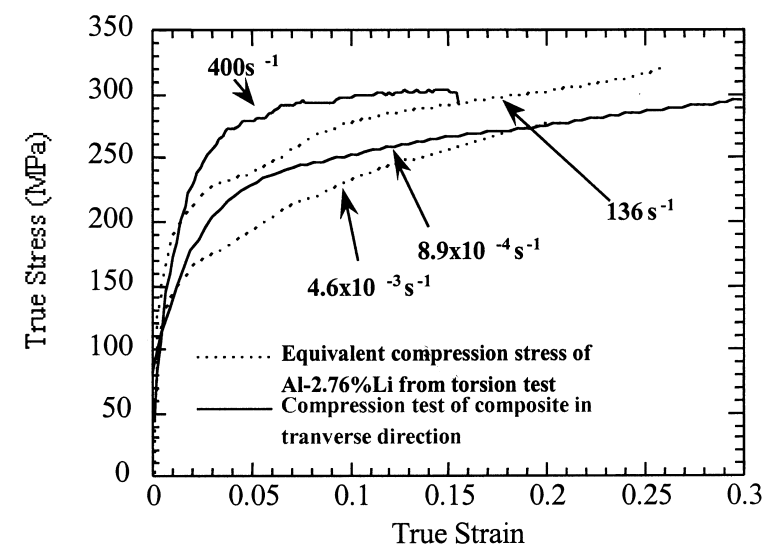

Fig. 6. Stress/strain curves of the FP fiber reinforced Al$3 \mathrm{wt} \% \mathrm{Li} \mathrm{MMC}$ in transverse direction and converted equivalent stress/strain curves of $\mathrm{Al}-2.76 \mathrm{wt} \% \mathrm{Li}$ [16]. alloy at equivalent strains of 5 and $1.5 \%$ are shown in Fig. 7 as a function of logarithm of strain rate. In the same figure, $5 \%$ strain flow stress values of the composite in the transverse direction are also shown for comparison.

In order to make comparison within the similar strain rate regime, only composite flow stress values from quasi-static to $600 \mathrm{~s}^{-1}$ (lowest strain rate achieved in SHPB) were included in Fig. 7. The flow stress points in Fig. 7 are fitted to the equation

$\sigma=\sigma_{0}+C \log \dot{\varepsilon}$

where $\sigma$ and $\sigma_{0}$ are the flow stress and flow stress at unit strain rate, respectively, $\dot{\varepsilon}$ is strain rate and $C$ is a parameter showing strain rate sensitivity. The $C$ values in Eq. (4) are found to be 8.3, 7.7 and 9.9 for composite and $\mathrm{Al}-2.76 \mathrm{wt} \% \mathrm{Li}$ at $5 \%$ strain and $\mathrm{Al}-2.76 \mathrm{wt} \% \mathrm{Li}$ at $1.5 \%$ strain, respectively. These values show similar trends in flow stresses at increasing strain rates for the composite in the transverse direction and the Al$2.76 \mathrm{wt} \% \mathrm{Li}$ alloy.

The interpolated flow stresses of the composite and $\mathrm{Al}-2.76 \mathrm{wt} \% \mathrm{Li}$ alloy at $5 \%$ strain were further used to interpolate the maximum stresses of the composite in the longitudinal direction at different strain rates. The flow stress values of the composite and Al-2.76wt $\% \mathrm{Li}$ were converted to shear stresses and accordingly the strain rates were converted to shear strain rates. The linear interpolation is shown in Fig. 8, both flow stress data sets showed a good fit to the maximum stress of the composite in the fiber direction. The slopes of the interpolations gave fiber misorientations of 2.12 and $1.44^{\circ}$ which are less than those of polymeric composites but are again reasonable in view of the larger diameter $(\sim 20 \mathrm{~mm})$ and higher stiffness of the $\mathrm{FP}^{\text {(i) }}$ fibers.

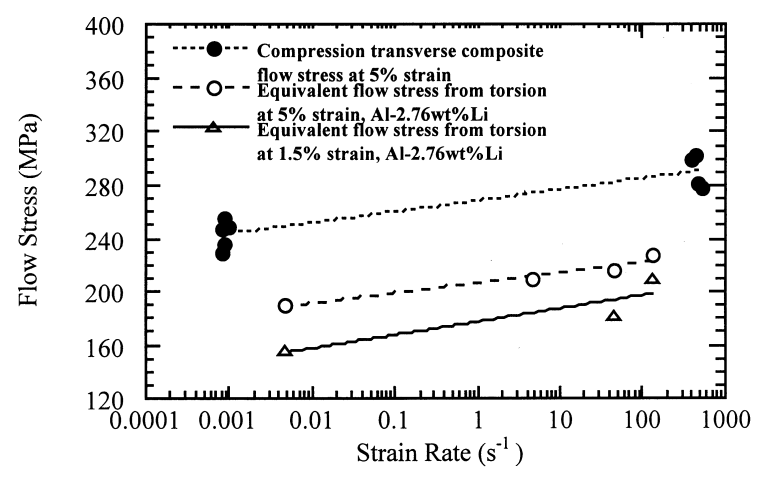

Fig. 7. Flow stress vs. log. strain rate behavior of FP reinforced $\mathrm{Al}-3 \mathrm{wt} \% \mathrm{Li}$. 


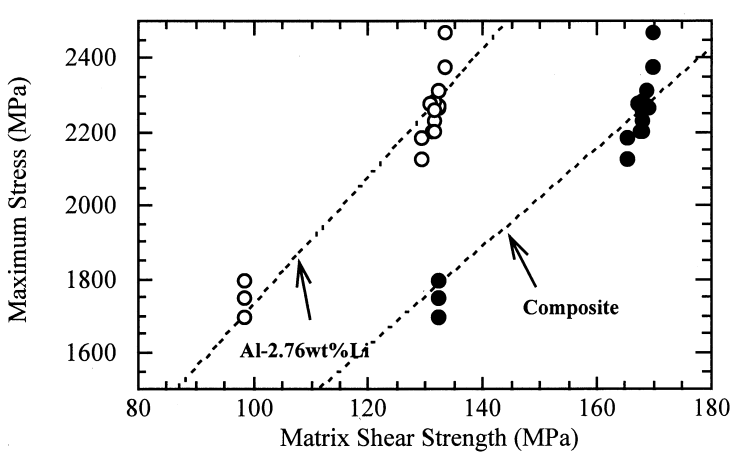

Fig. 8. Linear interpolation of the predicted matrix shear strength from composite and $\mathrm{Al}-2.76 \mathrm{wt} \% \mathrm{Li}$ alloy torsion data [16] and maximum stress FP fiber reinforced $\mathrm{Al}-3 \mathrm{wt} \% \mathrm{Li}$ MMC in longitudinal direction.

\section{Conclusions}

The mechanical response of an FP/Al composite has been determined in transverse and longitudinal directions in compression. It is found that the composite in the transverse direction exhibited strain rate sensitivity of the flow stress and maximum stress within the studied strain rate range. The flow stress rate sensitivity in this direction was found to be similar to the monolithic alloy rate sensitivity determined from previous work. The increased rate sensitivity in the longitudinal direction is well described in terms of strain rate dependent fiber buckling stress which scaled with matrix shear stress.

\section{References}

[1] Güden M, Hall IW. J Mater Sci 1998;33:3285.

[2] Evans AG, Adler WF. Acta Metall 1978;26:725.

[3] Pigot MR, Harris B. J Mater Sci Eng 1980;15:2523.

[4] Jelf PM, Fleck NA. J Composite Mater 1992;26:2706.

[5] Kyriakides S, Arseculeratne R, Perry EJ, Liechti K. Int J Solid Structures 1995;32:689.

[6] Sourtis C, Tenchev R. Sci Eng Comp Mater 1995;4:28.

[7] Moran PM, Liu XH, Shih CF. Acta Metall Mater 1995;43:2943.

[8] Poulsen JS, Moran PM, Shih CF, Byskov E. Mech Mater 1997;25:67.

[9] Fleck NA, Shu JY. J Mech Phys Solid 1995;43:1887.

[10] Vogler TJ, Kyriakides S. Acta Mater 1997;45:2443.

[11] Rosen BW. In: Fiber composite materials. Metal Park, OH: ASM, 1964. p. 37.

[12] Argon S. In: Treatise on materials science and technology, vol. 1. New York: Academic Press, 1972.

[13] Budiansky. Computers Struct. 1983:16:3.

[14] Lankford J. J Mater Sci 1995;30:4343.

[15] Chiem CY, Lee WS, Meyer P. J de Phys 1988;49:C3-19.

[16] Kobayashi S. Ph.D. Thesis. University of Reading, UK. 